\title{
THE MAJOR POLYPEPTIDES OF THE NUCLEAR PORE COMPLEX
}

\author{
GEORG KROHNE, WERNER W. FRANKE and ULRICH SCHEER
}

Division of Membrane Biology and Biochemistry, Institute of Experimental Pathology, German Cancer Research Center, D-6900 Heidelberg, Federal Republic of Germany

\begin{abstract}
SUMMARY
Nuclear envelopes of maturing oocytes of various amphibia contain an unusually high number of pore complexes in very close packing. Consequently, nuclear envelopes, which can be manually isolated in great purity, provide a remarkable enrichment of nuclear pore complex material, relative to membranous and other interporous structures. When the polypeptides of nuclear envelopes isolated from oocytes of Xenopus laevis and Triturus alpestris are examined by gel electrophoresis, visualized either by staining with Coomassie blue or by radiofluorography after in vitro reaction with $\left[{ }^{3} \mathrm{H}\right]$ dansyl chloride, a characteristic pattern is obtained (10 major and 15 minor bands). This polypeptide pattern is radically different from that of the nuclear contents isolated from the same cell. Extraction of the nuclear envelope with high salt concentrations and moderately active detergents such as Triton X-100 results in the removal of membrane material but leaves most of the non-membranous structure of the pore complexes. The dry weight of the pore complex (about 0.2 femtograms) remains essentially unchanged during such extractions as measured by quantitative electron microscopy. The extracted preparations which are highly enriched in nuclear pore complex material contain only two major polypeptide components with apparent molecular weights of 150000 and 73000 . Components of such an electrophoretic mobility are not present as major bands, if at all, in nuclear contents extracted in the same way. It is concluded that these two polypeptides are the major constituent protein(s) of the oocyte nuclear pore complex and are specific for this structure. When nuclear envelopes are isolated from rat liver and extracted with high salt buffers and Triton X-100 similar bands are predominant, but two additional major components of molecular weights of 78000 and 66000 are also recognized. When the rat liver nuclear membranes are further subfractionated material enriched in the 66000 molecular weight component can be separated from the membrane material, indicating that this is relatively loosely associated material, probably a part of the nuclear matrix. The results suggest that the nuclear pore complex is not only a characteristic ubiquitous structure but also contains similar, if not identical, skeletal proteins that are remarkably resistant to drastic changes of ionic strength as well as to treatments with detergents and thiol reagents.
\end{abstract}

The nuclear pore complex is a characteristic and ubiquitous structure of eukaryotic cells which provides a gateway for the nucleocytoplasmic exchange of molecules and particles and its control. This highly ordered complex is characterized by the association of distinct non-membranous structures with the pore orifice of the nuclear membrane proper [1-6]: (i) the outer and inner annulus, each containing eight globular subunits arranged in radial symmetry, (ii) eight cones of similarly dense material which centripetally project from the pore wall and are arranged in the same symmetry, and (iii) a central rod- or pluglike element of variable size and frequency. In addition, ordered fibril arrays occur in the pore complex and cytoplasmic and nuclear fibrils often are also connected with the annular and central components [2-8]. Information on the composition of these membrane-associated pore complex structures is scanty. Several authors have attempted to separate the membrane-asso- 
ciated pore complex components from the membrane material proper and to subfractionate isolated nuclear membranes by treatment with ultra-sonication [for refs see $5,9]$, high salt concentrations, and/or moderately active detergents such as Triton X100 [7, 10-14]. Although such experiments have been successful in the relative enrichment of pore complex material they invariably have resulted in fractions of pore complex structures that are still present in large aggregates, with the pore complex residues being connected by interporous sheet-like (lamina) or fibrillar structures. In most of these studies somatic cells such as hepatocytes and avian erythrocytes were used and the proportion of such interporous material retained in such residual material was relatively high, obviously a consequence of the low pore density present in these nuclei. Therefore, such preparations of pore complex- lamina- nuclear matrix remnants from somatic cells are difficult to interpret as to the correlation of the specific structures with the polypeptide components identified by gel electrophoresis. The picture obtained has been further complicated by the finding that somatic nuclei contain a fibrillar network ("nuclear matrix", for synonyms see refs quoted below) that is resistant to extractions with low and high salt buffers, nucleases, and moderate detergents and shows an enrichment of some polypeptides of similar sizes as those found in the nuclear membrane-derived residue fractions mentioned above $[8,15-23]$. Compared with the somatic nuclei used in the studies mentioned above, the nuclear envelope of the giant nucleus of the amphibian oocyte in mid-to-late stages of oogenesis offers some crucial advantages: (1) It contains a much higher number of pore complexes (ca $4 \times 10^{7}$ per nucleus) in very close packing $[3,4,24,25]$. (2) It is associated neither with chromatin nor with a "fibrous lamina" as described in various other cells [for review see 26]. And (3), it can be manually isolated in very high purity [24]. Therefore, we have decided to isolate and to subfractionate the nuclear envelope from such oocytes and to examine the polypeptides of the pore complex-enriched subfractions, in comparison with the polypeptides enriched in the pore complex enriched fractions from rat liver tissue.

\section{MATERIAL AND METHODS}

Isolation and extraction of nuclei, nuclear contents and nuclear envelopes from oocytes of Xenopus laevis and

\section{Triturus alpestris}

Small ovary pieces were removed from anaesthetized animals $(0.1 \%$ MS 222; Serva Feinbiochemica, Heidelberg) and placed in modified Barth's saline solution buffered with $10 \mathrm{mM}$ HEPES ( $N$-2-hydroxyethyl piperazine $N^{\prime}$-2-ethanesulphonic acid/ $\mathrm{NaOH}, \mathrm{pH} 7.6$ [27] which contained $1 \%$ of an amino acid solution (MEM, 100×; Seromed, LS-Labor Service, Munich). Nuclei were manually isolated from stage $\mathrm{V}$ oocytes of $X$. laevis [39] under a dissecting microscope in the "5: 1 -medium" $(0.1 \mathrm{M} \mathrm{KCl}$ and $0.1 \mathrm{M} \mathrm{NaCl}$ in a ratio of $5: 1$, buffered with $10 \mathrm{mM}$ Tris- $\mathrm{HCl}$ to $\mathrm{pH} 7.2$ ). In some experiments oocyte nuclei were directly isolated and washed in $1.5 \mathrm{M} \mathrm{KCl}, 10 \mathrm{mM}$ Tris, $\mathrm{pH} 7.2$. For the isolation of nuclear membranes cleaned nuclei were transferred into " $5: 1$-medium" with additional 10 $\mathrm{mM} \mathrm{MgCl} 2$. Within 30-60 sec the nuclear content gelifies and forms an opaque and compact aggregate clearly retracted and separated from the nuclear envelope. By sucking the nucleus into a pipette with a bore diameter $(0.4 \mathrm{~mm})$ slightly narrower than the mean nuclear diameter (ca $0.5 \mathrm{~mm}$ ) the nuclear membrane is locally broken, and the gelified nucleoplasmic "ball" slips out [for details see 24]. The isolated nuclear envelopes were cleaned in fresh medium by repeated excursions through a small pipette and finally transferred into an Eppendorf reaction tube $(1.5 \mathrm{ml}$ size). Usually $20-50$ nuclear envelopes were pooled (except preparations for Coomassie blue-stained gels in which 300-500 nuclear envelopes were pooled) and sedimented for $4 \mathrm{~min}$ at $8000 \mathrm{~g}$ in a laboratory centrifuge (Eppendorf Gerätebau, Hamburg). The supernatant was withdrawn, and the pelleted nuclear membranes were either stored at $-20^{\circ} \mathrm{C}$ or extracted in solutions containing the salt and detergent concentrations indicated below. In each extraction step the nuclear membranes were resuspended by vortexing in $0.5 \mathrm{ml}$ extraction solution and then pelleted by centrifugation at $8000 \mathrm{~g}$ for $4 \mathrm{~min}$. At the end of each extraction series, the residual material was washed in 
$10 \mathrm{mM}$ Tris- $\mathrm{HCl}(\mathrm{pH}$ 7.2), pelleted, decanted, and stored at $-20^{\circ} \mathrm{C}$. All extractions were carried out at room temperature with one of the following solutions which were buffered to $\mathrm{pH} 7.6$ with $10 \mathrm{mM}$ Tris $-\mathrm{HCl}$ or $100 \mathrm{mM}$ sodium phosphate (a) $1 \%$ Triton X-100 (15 min); (b) $1.5 \mathrm{M} \mathrm{KCl}(45 \mathrm{~min})$; (c) $0.5,1.0$ or $1.5 \mathrm{M}$ $\mathrm{KCl}(45 \mathrm{~min})$ followed by $15 \mathrm{~min}$ extraction with $1 \%$ Triton X-100; (d) $0.5 \mathrm{M} \mathrm{KCl,} 1 \%$ Triton X-100 (30 $\min )$; (e) $1.5 \mathrm{M} \mathrm{KCl}, 1 \%$ Triton X-100 (30 min); $(f)$ $0.5 \mathrm{M} \mathrm{MgCl}_{2}, 1 \%$ Triton X-100 (30 min); $(g) 1.0$ or 2.0 M urea $(20 \mathrm{~min})$; $(h) 0.5,1.0$ or $2.0 \mathrm{M}$ guanidinium hydrochloride $(\mathrm{GH}, 15 \mathrm{~min})$; (i) $1.0 \mathrm{M} \mathrm{GH}, 1 \%$ Triton $\mathrm{X}-100(30 \mathrm{~min}) ;(j) 0.5 \mathrm{M} \mathrm{GH}, 0.5 \%$ Triton $\mathrm{X}-100$, $0.2 \%$ sodium deoxycholate $(20 \mathrm{~min}) ;(k) 1 \%$ sodium deoxycholate $(20 \mathrm{~min})$. Nuclear contents obtained from 20-50 nuclei were pooled by centrifugation, extracted in parallel with the nuclear membranes, and were stored at $-20^{\circ} \mathrm{C}$. Isolated nuclei and nuclear contents which were not subjected to the extraction procedures were directly collected in ice-cold $96 \%$ ethanol. After centrifugation for $5 \mathrm{~min}$ at $3000 \mathrm{~g}$ the pellets were dried under nitrogen and stored at $-20^{\circ} \mathrm{C}$. Nuclei and nuclear envelopes from maturing oocytes of the newt, Triturus alpestris, were isolated and purified with similar techniques $[3,12]$.

\section{Isolation of nuclei and nuclear membranes \\ from rat liver}

Nuclei and nuclear membranes from the liver tissue of young rats (Sprague-Dawley, both sexes) were isolated essentially according to previously described methods $[28,29]$. Inclusion of $n$-octanol and/or 2mercaptoethanol in the homogenization medium (medium A in ref. [29]) did not significantly influence the results described here. In an alternative procedure the filtrated homogenate was centrifugated for $10 \mathrm{~min}$ at $850 \mathrm{~g}$, the loosely packed crude nuclear pellet was resuspended in the same buffer and adjusted to $2.1 \mathrm{M}$ with $2.5 \mathrm{M}$ sucrose (in $10 \mathrm{mM}$ Tris- $\mathrm{HCl}, \mathrm{pH} 7.4,0.5$ $\mathrm{mM} \mathrm{MgCl} 2$ ). This suspension was then laid over a cushion (ca $12 \mathrm{ml}$ in a $34 \mathrm{ml}$ ultracentrifuge tube) and the whole was centrifuged at ca $100000 \mathrm{~g}$ for $60 \mathrm{~min}$. The nuclear pellet was usually purified by an additional cycle of resuspension and centrifugation in high sucrose concentration.

Nuclear membranes isolated according to the various modifications described previously [29] gave essentially similar results as to the pattern of the major polypeptides of the pore complex-enriched residues obtained after high salt and detergent treatment. Routinely, we used the following modification. Nuclei were washed once by resuspension and centrifugation (as above) in $0.4 \mathrm{M}$ sucrose $(10 \mathrm{mM}$ Tris- $\mathrm{HCl}, \mathrm{pH} 7.4$, $70 \mathrm{mM} \mathrm{KCl}$ ). The washed nuclei were resuspended in $0.1 \mathrm{mM}$ Tris-distilled water, $\mathrm{pH} 7.8$, using a Dounce homogenizer, and were pelleted after $5 \mathrm{~min}$ incubation by centrifugation at $3000 \mathrm{~g}$ for $5 \mathrm{~min}$. The swollen nuclei were then thoroughly resuspended in $0.3 \mathrm{M}$ sucrose (10 mM Tris- $\left.\mathrm{HCl}, \mathrm{pH} 7.4,0.2 \mathrm{mM} \mathrm{MgCl}_{2}\right)$, and pancreatic deoxyribonuclease (grade 1, Worthington Biochemicals, Freehold, New Jersey, USA) was added from a stock solution (same buffer) to a final concentration of $5 \mu \mathrm{g} / \mathrm{ml}$, corresponding to about 10 $\mu \mathrm{g} / \mathrm{mg}$ DNA. After digestion for $10 \mathrm{~min}$ at $30^{\circ} \mathrm{C}$ the same amount of DNAse stock solution was added and incubation was continued for another $10 \mathrm{~min}$. The reaction was stopped by addition of the eight-fold volume of cold $\left(0^{\circ}-5^{\circ} \mathrm{C}\right) 1.5 \mathrm{M} \mathrm{KCl}$ in $10 \mathrm{mM}$ Tris$\mathrm{HCl}(\mathrm{pH} \mathrm{7.4)}$. After extraction for $1 \mathrm{~h}$ under gentle stirring in the coldroom this suspension was centrifuged through a small cushion of $0.9 \mathrm{M}$ sucrose in 1.5 $\mathrm{M} \mathrm{KCl}$ (same buffer) for $1 \mathrm{~h}$ at about $40000 \mathrm{~g}$. The pellet obtained was resuspended with the use of a Potter-Elvehjem homogenizer in $0.3 \mathrm{M}$ sucrose $(0.2$ $\mathrm{mM} \mathrm{MgCl}_{2}$, same buffer), and this suspension was adjusted to $2.1 \mathrm{M}$ sucrose. It was then filled into ultracentrifuge tubes (ca $12 \mathrm{ml}$ per $34 \mathrm{ml}$ tube), overlaid by a sucrose gradient (same buffer) ranging from $2.1 \mathrm{M}$ to $1.2 \mathrm{M}$ sucrose, and this was centrifuged for $3 \mathrm{~h}$ (or overnight) at approx. $100000 \mathrm{~g}$ in the cold $\left(4^{\circ}-\right.$ $8^{\circ} \mathrm{C}$ ). Bands with mean peak buoyant densities in the range of $1.180-1.195 \mathrm{~g} \mathrm{~cm}^{-3}$ contained the nuclear membrane material and were collected using conventional techniques. A pellet observed at this step consisted mainly of nuclear matrix and nucleolar residues, and some histone-depleted chromatin-derived material ("first pellet"). The material collected from the band and pelleted (in $10 \mathrm{mM}$ Tris-buffer as above; $1 \mathrm{~h}$ at $100000 \mathrm{~g}$ ) was designated "nuclear membranes purified once". An aliquot of this material was directly resuspended in an excess amount of $1.5 \mathrm{M} \mathrm{KCl}$ solution (same buffer) containing $1 \%$ Triton $\mathrm{X}-100$, and this was stirred for $20 \mathrm{~min}$ at room temperature. Another aliquot was re-homogenized and purified once more in the floating gradient system described above, and the resulting material collected from the bands, which then appeared at slightly lower densities (the usual decrement was about $0.015 \mathrm{~g} \mathrm{~cm}^{-3}$ ), was pelleted as described above and designated "nuclear membranes purified twice". The pellet obtained in this second centrifugation contained membrane-detached fibrillar aggregates resembling intranuclear framework components and was designated "nuclear membranederived heavy material". These latter fractions were then also extracted in the $1 \%$ Triton X-100-containing high salt buffer described above. Material resistant to the combined detergent and high salt buffer treatment in the specific fractions was pelleted by centrifugation at $100000 \mathrm{~g}(1 \mathrm{~h}$, room temperature $)$ either directly or after exhaustive dialysis against $1 \mathrm{mM}$ Tris- $\mathrm{HCl}$ (pH 7.4) containing $0.1 \%$ sodium dodecylsulphate (SDS), with repeated changes of the dialysis buffer. In some experiments all solutions used had been adjusted to 3 or $5 \mathrm{mM}$ 2-mercaptoethanol or dithiothreitol.

\section{Polyacrylamide gel electrophoresis}

Proteins were solubilized in $20-40 \mu \mathrm{l}$ sample buffer (10 mM phosphate buffer, $\mathrm{pH} 7.2,5 \%$ SDS, $10 \%$ 2 -mercaptoethanol, $10 \%$ glycerol) at $100^{\circ} \mathrm{C}$ for 1 to 5 min either directly or after radioactive labelling with tritiated dansyl chloride [30]. After incubation with 25 $\mu \mathrm{Ci}\left[{ }^{3} \mathrm{H}\right]$ dansyl chloride $(18 \mathrm{Ci} / \mathrm{mmol}$; Radiochemical Centre, Amersham) the reaction was terminated by adding $25 \mu \mathrm{g}$ chymotrypsinogen and ice-cold trichloroacetic acid (TCA) to a final concentration of $13 \%$. In some experiments $25 \mu \mathrm{g}$ bovine serum albumin or 

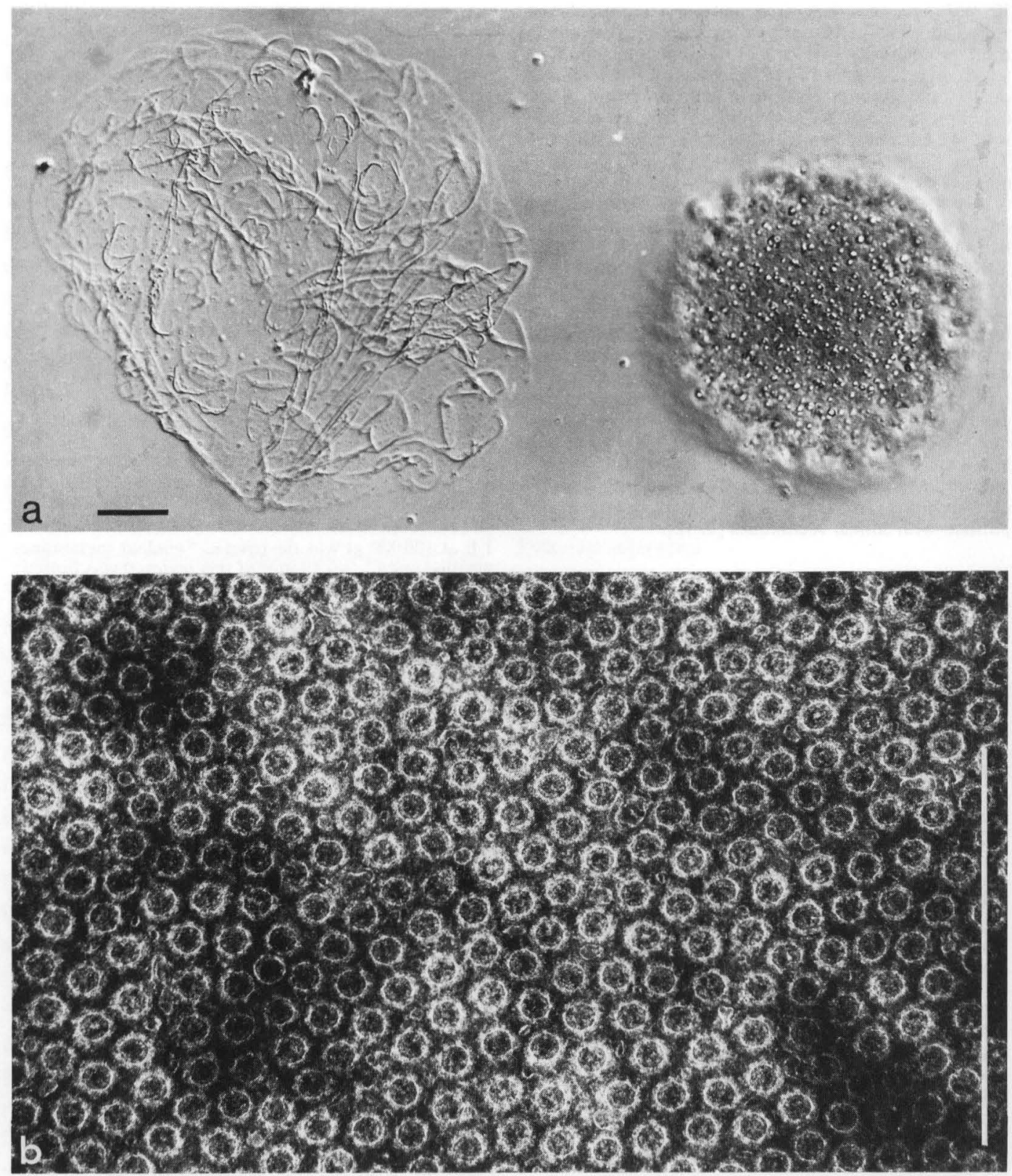

Fig. 1. Separation of nuclear envelope and nuclear contents of maturing $X$. laevis oocytes and appearance of the isolated nuclear envelopes. An isolated nucleus is ruptured, the nuclear envelope "ghost" $(a, l e f t)$ and the gelified nuclear content $(a$, right) are manually separated. (a) Presents a light micrograph taken with interference contrast optics. Electron micrographs of negatively stained preparations $(b)$ illustrate the high number and the dense packing of the pore complexes of such nuclear envelopes. Bars denote $100 \mu \mathrm{m}(a)$ and $1 \mu \mathrm{m}(b)$. 
phosphorylase- $a$ was used for the co-precipitation instead of chymotrypsinogen. After storage for $1 \mathrm{~h}$ at about $4^{\circ} \mathrm{C}$ the precipitate was pelleted $(4 \mathrm{~min}, 8000 \mathrm{~g})$ and washed five times each with $150 \mu \mathrm{l}$ ice-cold $10 \%$ TCA. The final pellet was solubilized in $20 \mu \mathrm{l}$ sample buffer by incubation for $1 \mathrm{~h}$ at $60^{\circ} \mathrm{C}$.

$7.5 \%$ and $10 \%$ acrylamide slab gels were prepared and run as described by Laemmli [31], 20\% gels as described by Thomas \& Kornberg [32]. Stacking gels were $3.9 \%$ [31]. Mol. wt standards used were phosphorylase- $a$ (94000), bovine serum albumin (67000), actin from rabbit skeletal muscle (42000), chymotrypsinogen (25000), and cytochrome $c(13000)$. In some experiments tubulin from porcine brain (55000) and myosin from rabbit skeletal muscle (approx. 220000 ) were also included as references. Apparent mol. wt of separated polypeptide bands were determined according to Weber \& Osborn [33]. Gels were fixed and stained in a solution containing $20 \%$ methanol, $7 \%$ acetic acid, $0.6 \%$ TCA and $0.025 \%$ Coomassie blue ( $1 \mathrm{~h}$ at $70^{\circ} \mathrm{C}$ or $12 \mathrm{~h}$ at room temperature) and were destained by repeated washing in $7 \%$ acetic acid. In some cases gels were stained by the periodic acid-Schiff reaction to detect glycoprotein [34]. For autoradiofluorography the fixed and destained gels were dehydrated in dimethylsulphoxide (DMSO), soaked in a solution of 2,5-diphenyloxazole in DMSO, dried and exposed to Kodak X-ray film (Kodak RP Royal "X-Omat") at $-70^{\circ} \mathrm{C}$ [35]. Gels and developed $X$-ray films were scanned at $550 \mathrm{~nm}$ in a Gilford spectrophotometer equipped with a linear gel transport device. Areas under defined peaks were determined with an image analyzing system MOP-AM-01 (Kontron Messgeräte, Eching/Munich).

\section{Light and electron microscopy}

Isolated nuclear envelopes were placed, without or after one of the extraction procedures described above, in lucite micro centrifuge chambers [36] and centrifuged for $10 \mathrm{~min}$ at $3000 \mathrm{~g}$ onto pioloform-coated electron microscopic grids. Grids were either observed directly or were immersed for $2-5 \mathrm{~min}$ in $2.5 \%$ glutaraldehyde in $50 \mathrm{mM}$ sodium cacodylate buffer ( $\mathrm{pH} 7.2$ ) with $50 \mathrm{mM} \mathrm{KCl}$ and $2.5 \mathrm{mM} \mathrm{MgCl}_{2}$, then washed with the same buffer, post-fixed for 2-5 min with $2 \% \mathrm{OsO}_{4}$, rinsed in distilled water, and negatively stained with $2 \%$ phosphotungstic acid adjusted with $\mathrm{NaOH}$ to $\mathrm{pH}$ 7.2. Ultrathin sections were obtained as described elsewhere [24]. Electron micrographs were routinely taken at $60 \mathrm{kV}$ (incl. dry mass determinations) or $80 \mathrm{kV}$. All light micrographs were taken with the inverted microscope Zeiss IM 35.

For dry mass determinations treated or untreated isolated nuclear envelopes were centrifuged onto carbon-coated grids, rinsed briefly in distilled water and were air-dried without fixation or staining. As a control untreated nuclear envelopes were fixed with $2 \%$ glutaraldehyde (see above), stepwise dehydrated in graded acetone solutions, and dried using the critical point method. In some experiments isolated nuclear envelopes were treated with the high salt buffer or the detergent or both together after attachment to the carbon-coated grid. Mass determinations of single nuclear pore complexes were carried out in the labora- tory of Dr G. F. Bahr (Department of Cellular Pathology, Armed Forces Institute of Pathology, Washington DC, USA). As standards latex beads of different diameters and a variety of viruses were used ([37]; for further technical details see there and [38]). Dry mass values of nuclear pore complexes were determined using identical areas of interporous regions of the partly distorted envelopes as well as of regions of the supporting film. The two references did not show significant differences which reflects the low dry mass concentration of these layers. For quantitation of percentages of total dry weight per pore complexes and interporous regions the image analyzing system (see above) was used.

\section{RESULTS}

\section{Morphology and dry weight of the pore complex after different extraction procedures}

The typical result of a manual separation of the nuclear envelope from the gelified nuclear contents of a maturing Xenopus laevis oocyte (stage V of ref. [39]) is shown in fig. $1 a$. The further purification of the envelope by washing in medium as well as the final purity of the fraction has been described in detail in a previous publication [24]. Fig. $1 b$ illustrates the high pore complex density and the pore complex architecture as revealed in negatively stained nuclear envelopes (45-60 pores per $\mu \mathrm{m}^{2}$; cf $[3,4,24,25])$. The granular and fibrillar components of the pore complex are well preserved. In negatively stained preparations it is noted that, with prolonged times of incubation in the isolation medium and/ or in the staining solution, the non-membranous constituents of the pore complex became infiltrated by the staining material and are less readily recognized [3]. When the nuclear envelopes are treated with high salt buffer (30-60 min in $10 \mathrm{mM}$ Tris- $\mathrm{HCl}$, $\mathrm{pH} 7.2$, containing $1.5 \mathrm{M} \mathrm{KCl}$ or $1.0 \mathrm{M}$ $\mathrm{KCl}$, or $0.5 \mathrm{M} \mathrm{MgCl}_{2}$ ) most of the pore complex material is retained both in the presence and absence of thiol reagents such as $5 \mathrm{mM}$ 2-mercaptoethanol and dithiothrei- 


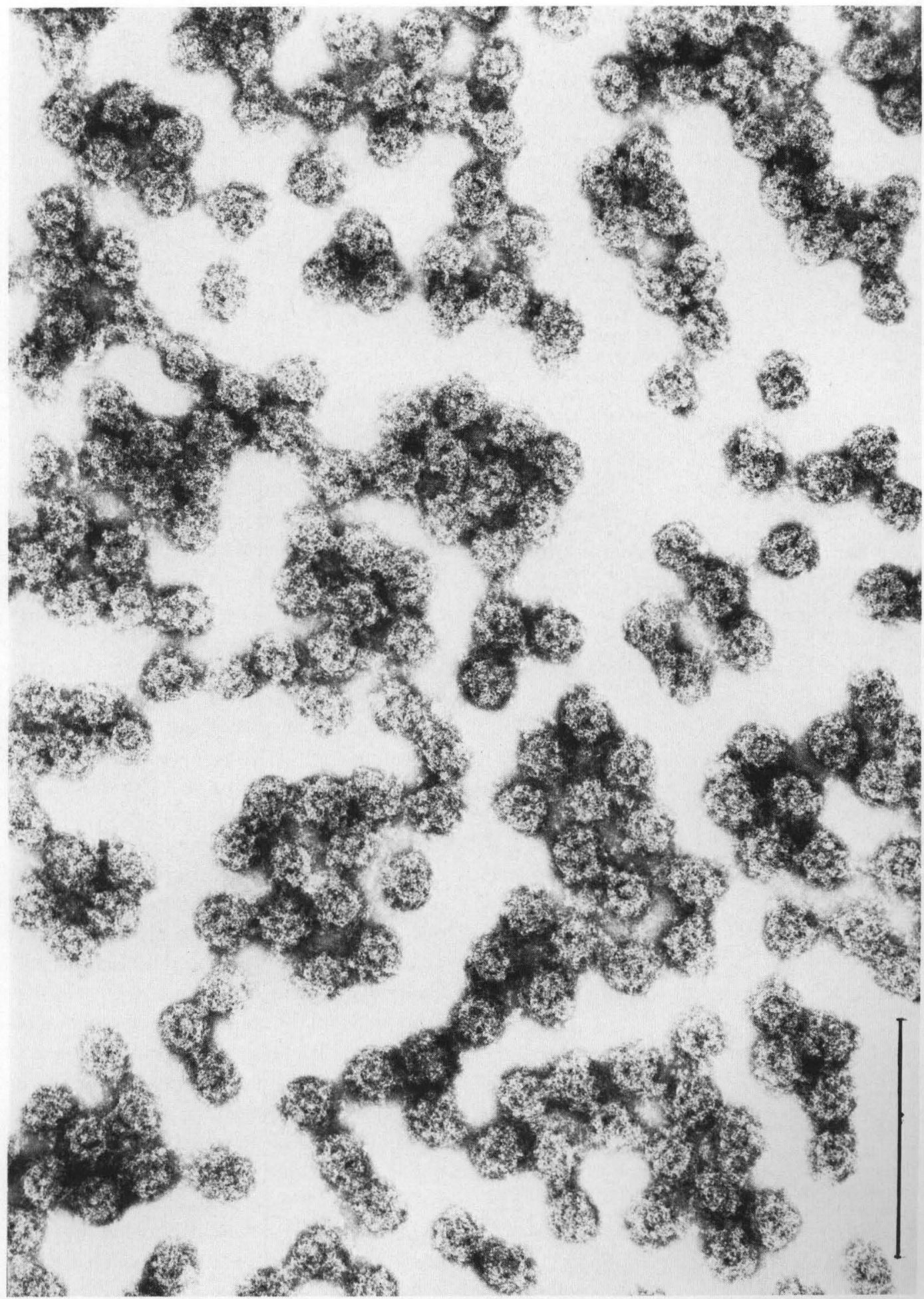


tol. Figs 2 and 3 show preparations of nuclear envelope residues obtained after combined treatment with high salt buffer and saturating amounts of detergent, Triton X100 , as revealed after negative staining and ultrathin sections. The non-membranous pore complex structures are well preserved, and details of internal architecture such as central granules and annular subunits are frequently recognized, though usually with somewhat reduced distinctiveness of the individual subcomponents. Many of the residual pore complexes appear to be interconnected by fine interporous fibrils (fig. 3 ; cf [12]). The appearance of such preparations is essentially identical whether $1.0 \mathrm{M}$ $\mathrm{KCl}, 1.5 \mathrm{M} \mathrm{KCl}$, or $0.5 \mathrm{M} \mathrm{MgCl}_{2}$ has been used, and whether thiol reagents have been included or not. No significant change is observed if the treatment with the high salt buffer and the detergent is not sequential but simultaneous.

When the total dry weight per pore complex is determined in unstained preparations, fixed or unfixed, by quantitative electron microscopy it is found that most of the pore complex mass is retained during treatment with high salt concentrations and Triton X-100 (table 1). The mean value of the dry weight of the total nuclear pore complex as determined in these preparations (about 0.2 femtogram) is markedly lower than that reported by DuPraw ([40]; 0.5 femtograms in honeybee embryo nuclei). From these measurements it can be estimated that the residual pore complex protein per nucleus is about $8 \mathrm{ng}$, which rep-

Fig. 2. Survey electron micrograph of a negatively stained preparation showing the nuclear pore complex-enriched fraction of $X$. laevis oocytes obtained after treatment with high salt concentrations and detergent. The sequential extraction with buffers containing $1.5 \mathrm{M} \mathrm{KCl}$ and $1 \%$ Triton removes most of the interporous membrane material but leaves most of the pore complex structure intact. Bar denotes $0.5 \mu \mathrm{m}$. resents less than $0.1 \%$ of the total nuclear protein [cf 41].

As can be seen in figs 2 and 3 the by far predominant portion of the stainable material of the high salt- and detergent-extracted preparations is contained in the non-membranous pore complex residues. Estimations from quantitative electron microscopy of the electron dense mass contained in pore complexes and interporous fibrils of extracted nuclear envelopes show that a very high proportion (usually more than $85 \%)$ of the dry mass is contained in the pore complexes.

Residual pore complex structures are also observed after treatment with buffers containing $2 \mathrm{M}$ urea or $1 \mathrm{M}$ guanidinium hydrochloride (results not shown here), irrespective of a preceding extraction with high salt buffer and detergent. However, incubation in solutions containing $3 \mathrm{M}$ guanidinium hydrochloride or $6 \mathrm{M}$ urea or more results in progressive disintegration and solubilization of the pore complex material.

When nuclear envelopes from maturing oocytes of the urodelan amphibia Triturus alpestris, T. cristatus, and Pleurodeles waltli are isolated and extracted under the same conditions (high salt concentrations, detergents) essentially identical observations are made [ $\mathrm{cf} 12]$.

\section{Polypeptide components of isolated} nuclear envelopes from amphibian oocytes and of pore complex-enriched subfractions

Fig. 4 shows (in slot 4 ) the gel electrophoretic separation of the polypeptides of individually and manually isolated and washed, non-extracted nuclear envelopes from maturing oocytes of $X$. laevis, as revealed after staining with Coomassie blue, in comparison with the polypeptide pat- 

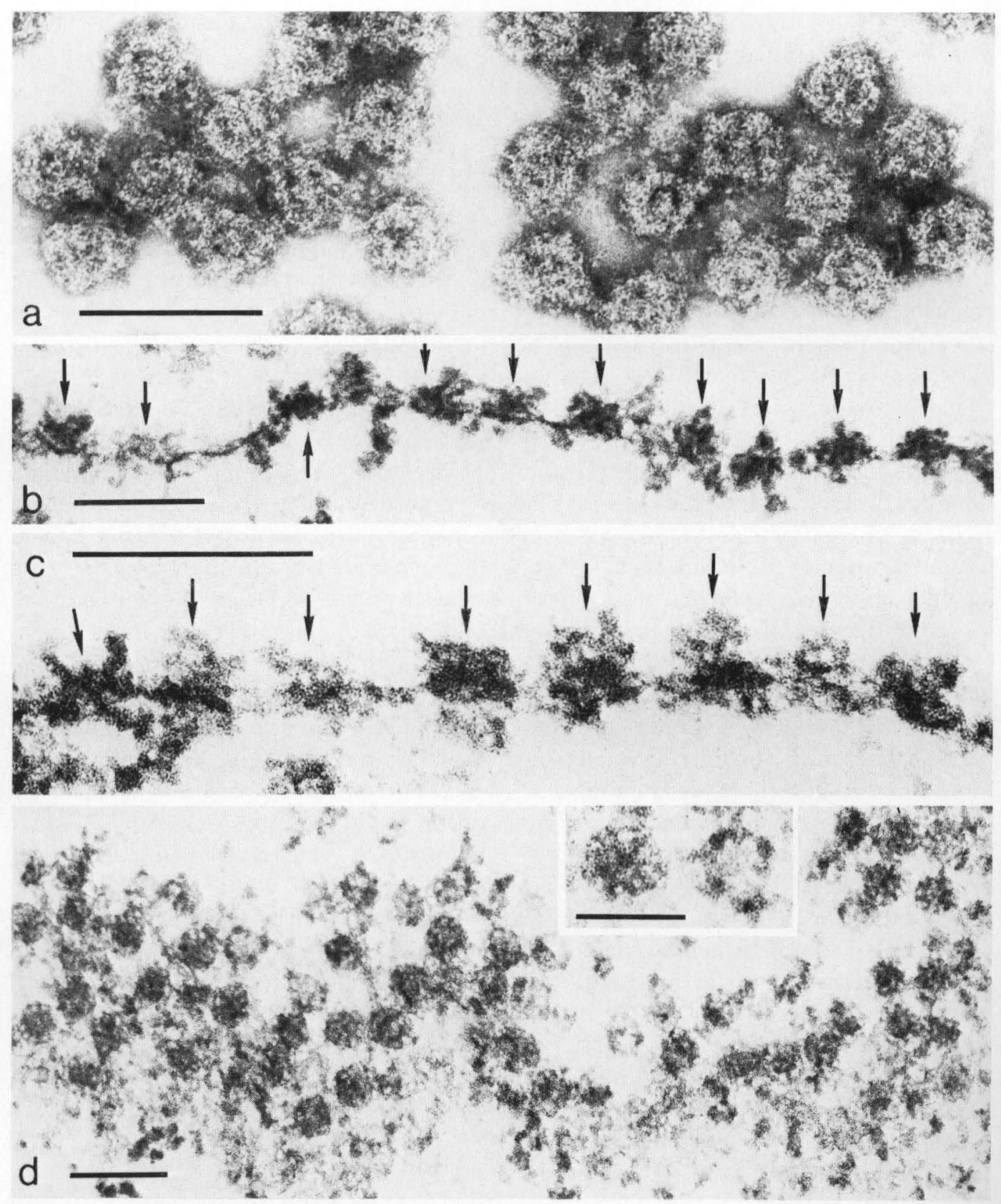

Fig. 3. Details of appearance of nuclear envelope residues obtained after treatment with high salt buffer and Triton (same treatment as shown in fig. 2) as revealed in negatively stained $(a)$ and sectioned $(b-d)$ preparations. Most of the pore complex such as annular subunits, centripetal tips, and central granule, is not soluble in $1.5 \mathrm{M} \mathrm{KCl}$ and $1 \%$ Triton as demonstrated in cross sections $(b, c)$ and grazing sections
( $d$, the inset shows, at higher magnification, two pore complexes sectioned at different levels). In such nuclear envelope residues the major part of the electrondense material is located in the pore complexes (arrows). Note also the pore complex-connecting material [cf ref. 12]. Bars denote $0.2 \mu \mathrm{m}(a-d)$ and $0.1 \mu \mathrm{m}$ (inset in $d$ ). 
Table 1. Dry mass determinations ${ }^{a}$ of individual nuclear pore complexes of mature Xenopus laevis oocytes by quantitative electron microscopy

Dry mass ${ }^{a}$

(attograms)

1. Unfixed nuclear envelope, air-dried

Untreated

Treated with $1.5 \mathrm{M} \mathrm{KCl}$

Treated with $1.5 \mathrm{M} \mathrm{KCl}$, followed

by $1 \%$ Triton

Treated with $0.5 \mathrm{M} \mathrm{MgCl}_{2}$ and

$1 \%$ Triton

$203(161-258)$

$219(182-264)$

$208(167-259)$

$176(144-216)$

2. Unfixed nuclear envelope, dried with the critical point method

Untreated

$245(161-305)$

3. Nuclear envelope fixed with glutaraldehyde, and dried with the critical point method

Untreated

$259(214-313)$

$a$ All values are given in attograms $\left(10^{-18} \mathrm{~g}\right)$ and represent the median of the mass distribution of at least 100 pore complexes. Parentheses denote the deviation in the form of the "sextile differences", i.e. the range between the percentiles 16 and 84 . This means that $68 \%$ of all measured values are within this range.

terns of total nuclei and of the separated gelified nuclear contents. In all experiments a typical nuclear envelope polypeptide pattern is obtained which contain 10 major and 15 minor bands (fig. 4, slot 4; table 2). The pattern of the nuclear envelope polypeptides is clearly different from the pattern of the polypeptides of the nuclear contents. For example, a series of components of $X$. laevis oocyte nuclei such as one with an apparent molecular weight of 165000 , a closely spaced triplet between 96000 and 104000 molecular weight, three bands with mobilities corresponding to 47000,45000 and 44000 apparent molecular weight, as well as a very prominent component comigrating with actin [cf 41, 42] are absent or represent only minor bands in the nuclear envelope material. For our studies it has been especially important to compare, in the same gel
Table 2. Estimated apparent molecular weights of the major polypeptides ${ }^{a}$ of manually isolated, non-extracted nuclear envelopes from maturing Xenopus laevis oocytes

\begin{tabular}{ll}
\hline Major band & $\begin{array}{l}\text { Approx. apparent } \\
\text { mol.wt }\end{array}$ \\
\hline 1 & 170000 \\
2 & 150000 \\
3 & 128000 \\
4 & 125000 \\
5 & 120000 \\
6 & 105000 \\
7 & 90000 \\
8 & 73000 \\
9 & 59000 \\
10 & 42000 \\
\hline
\end{tabular}

${ }^{a}$ Bands revealed in Coomassie blue-stained polyacrylamide gels were classified as mahor (denoted by the long bars in fig. 4) and minor components.

electrophoretic system, the mobility of the nuclear envelope polypeptides as revealed by staining with Coomassie blue (fig. 4, slot 4) with the pattern of the $\left[{ }^{3} \mathrm{H}\right]$ dansylated polypeptides as revealed in radiofluorographs (fig. 4, slot 5). There is excellent correspondence of the pattern of polypeptides revealed with either procedure, except for the uppermost band (approx. mol. wt ca 170000 ) which is either degraded during the dansylation procedures or, what seems very unlikely, refolds upon denaturation in SDS-buffer in a way that makes it less susceptible for dansylation (this polypeptide, however, is well dansylated in the original non-denatured state; G. Krohne, unpublished finding). Therefore, the labelling of polypeptides in vitro with $\left[{ }^{3} \mathrm{H}\right]$ dansyl chloride has been used in most of the later experiments since it allows a reduction of the number of isolated nuclear envelopes required per routine analysis by a factor of 10 to 20 . It is also noteworthy that the nuclear envelopes do not contain considerable amounts of polypeptides of low molecular 


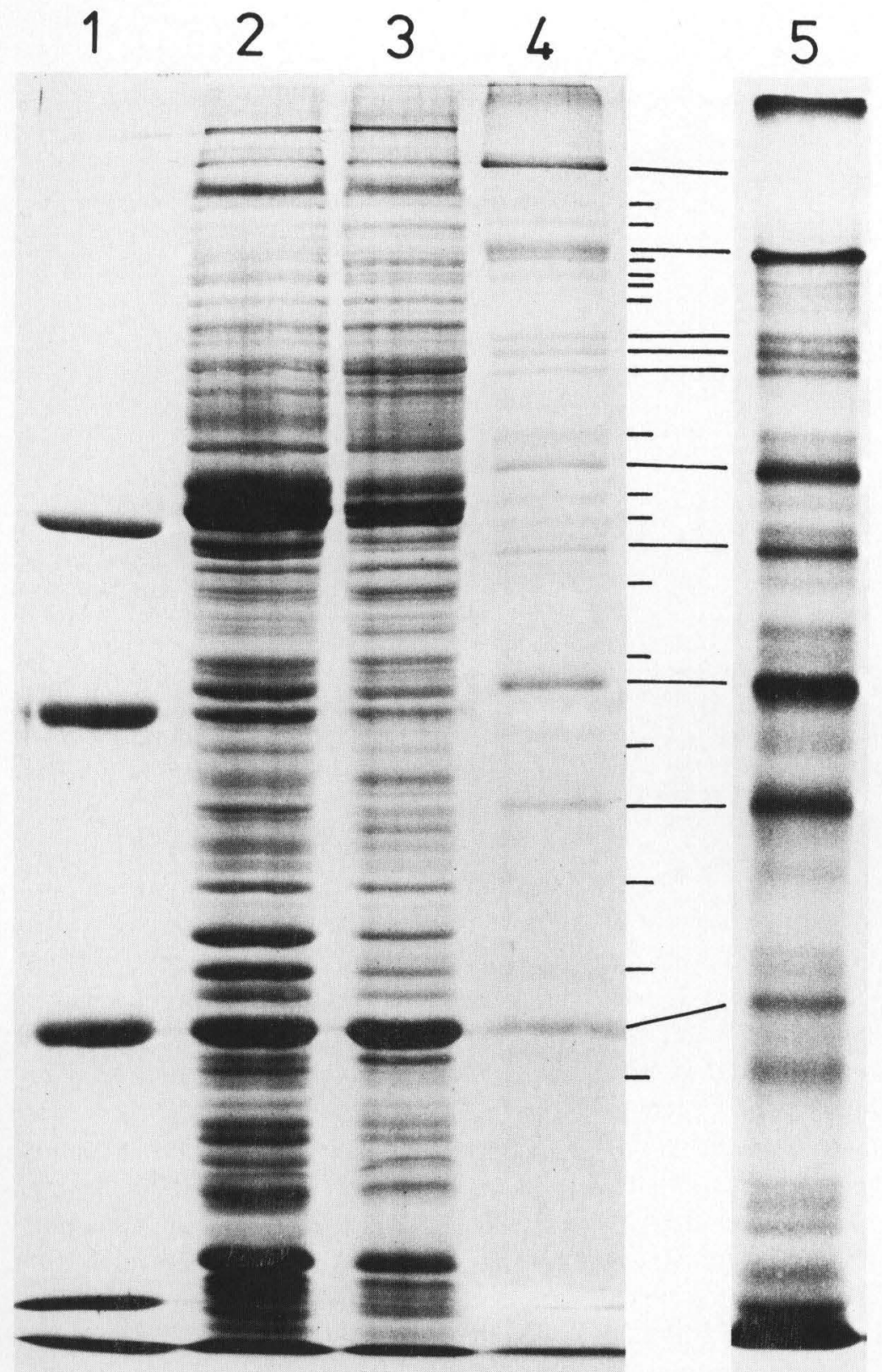

Fig. 4. Gel electrophoretic analysis of polypeptides of isolated nuclei and subnuclear fractions from $X$. laevis oocytes. Proteins of 30 nuclei (slot 2), 60 (slot 3) gelified nuclear contents, and 414 (slot 4) or 23 (slot 5) manually isolated nuclear envelopes have been separated on SDS-containing $10 \%$ polyacrylamide slab gels. Proteins are either stained with Coomassie blue (slots 1-4) or are identified by radiofluorography (slot 5). The pattern of the Coomassie blue-stained nuclear envelope polypeptides corresponds to that revealed in the fluorograph (long bars between slots 4 and 5 denote major bands, minor components are denoted by the short marks). The nuclear envelope pattern, however, is clearly different from that of total nuclei or nuclear contents. Molecular weight standards shown here (slot 1) are (from top to the bottom) phosphorylase- $a$, bovine serum albumin, actin, and chymotrypsinogen. 


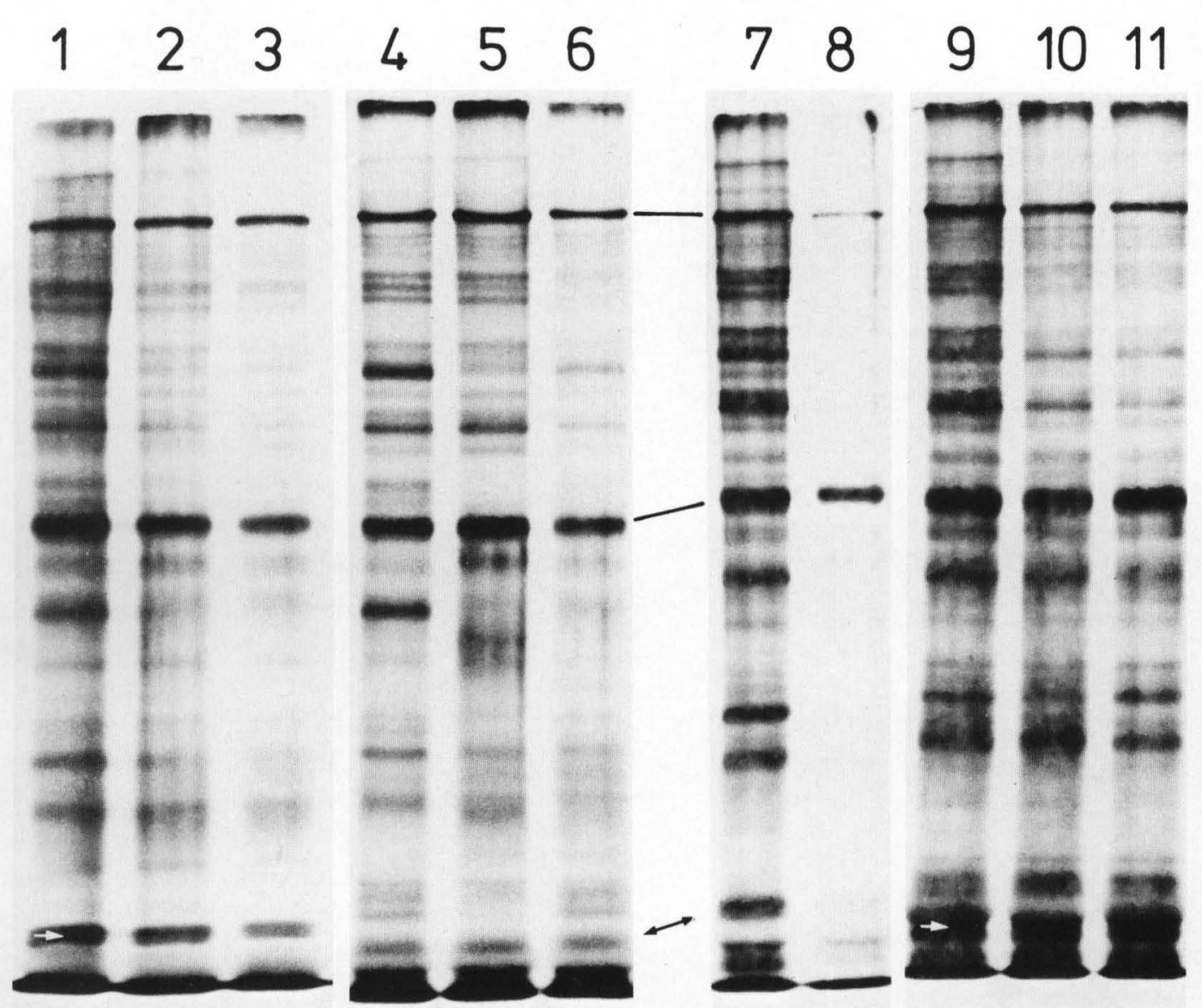

Fig. 5. Radiofluorographs of the dansylated polypeptides of the nuclear envelope residues ( $X$. laevis oocytes) obtained after various extraction procedures and separated by gel electrophoresis on $10 \%$ acrylamide gels. Slots $1-3$, slots $4-6$, slots 7 and 8 , and slots 9-11 represent the specific results of parallel experiments using oocytes of the same frog. Slots 1, 4, 7 and 9 show the polypeptides present in untreated nuclear envelopes (22-25 envelopes each). After extraction with buffers containing $1.5 \mathrm{M} \mathrm{KCl}$ (slot 10) or $1 \%$ Triton (slot 5 ) some polypeptide bands disap- pear but most components are retained. After combined sequential (slots $2,3,6$ ) or simultaneous (8) extraction with buffers containing high salt concentrations $(1.5 \mathrm{M} \mathrm{KCl}$ ) and $1 \%$ Triton (equivalent amounts from $41-45$ nuclear envelopes per slot) a drastic simplification of the pattern is noted, and two bands with apparent mol. wt of 150000 and 73000 predominate (denoted by the horizontal bars). Proteins retained after extraction with buffers containing $2.0 \mathrm{M}$ urea are shown in slot 11. The arrows denote the position of dansylated chymotrypsinogen. weights, including histones (fig. 4 , slot 4 ; fig. 6, slot 4).

Among the major polypeptides found in isolated non-extracted nuclear envelopes from $X$. laevis oocytes two components are of special interest. This is one component (band 2) with a relative electrophoretic mobility corresponding to an apparent mean molecular weight of about 150000 (a range of 145000 to 162000 was found in 20 different experiments), and a smaller component (band 8) with an apparent molecular weight of about 73000 . As estimated from densitograms, the former component represents about $9 \%$ of the total Coomassie blue-stained nuclear envelope polypeptides 


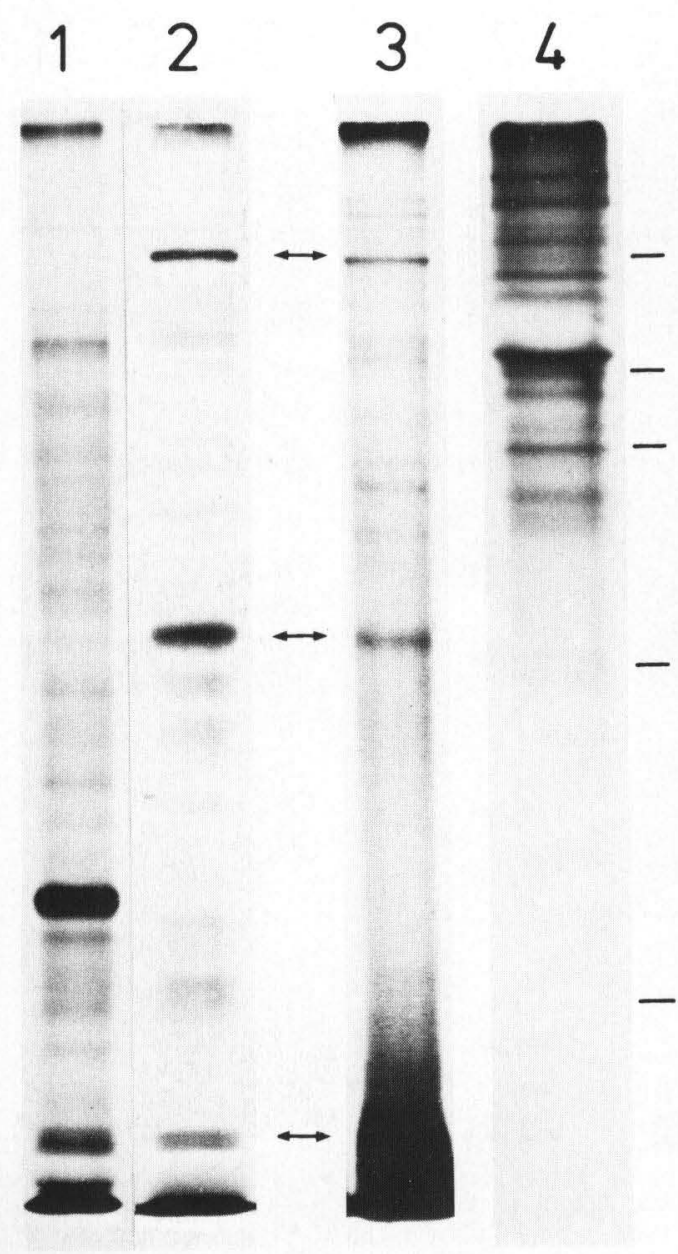

Fig. 6. Comparison of the polypeptide patterns of native and residual fractions of nuclear contents and nuclear envelopes from oocytes of $X$. laevis and $T$. alpestris. Dansylated polypeptides have been separated on $10 \%$ (slots 1-3) and 20\% (slot 4) acrylamide gels. The polypeptide pattern of nuclear envelopes extracted with high salt concentrations and Triton (slot 2 , sequential extractions with buffers containing 1.5 $\mathrm{M} \mathrm{KCl}$ and $1 \%$ Triton) is clearly different from that of the gelified nuclear contents after treatment with high salt buffer and Triton (slot 1, equivalent to the residues of 43 nuclei). In non-extracted nuclear envelopes ( $X$. laevis), polypeptides with mobilities corresponding to mol. wt below 30000 are virtually absent as demonstrated by gel electrophoresis in $20 \%$ gel (slot 4; bars denote, from top to the bottom, the position of phosphorylase- $a$, bovine serum albumin, actin, chymotrypsinogen, and cytochrome $c$ in this gel system). The two major polypeptide bands of residual pore complex structures are also found in isolated nuclear envelopes from $T$. alpestris oocytes after sequential extractions with high salt concentrations (1.5 M and about $6 \%$ of the total radioactivity of the $\left[{ }^{3} \mathrm{H}\right]$ dansylated material recovered in the gel, whereas the smaller component contains $11 \%$ and $12 \%$ of the stain and the radioactivity, respectively. Both components are only minute portions of the total nuclear protein. These two components, however, are the only prominent bands recognized in gel electrophoretic separations of the polypeptides of the pore complexenriched preparations obtained after sequential extraction of isolated nuclear envelopes with high salt concentrations and Triton X-100 (fig. 5, slots 1-6). Treatments with either Triton X-100 or high salt buffers alone does not result in such specific enrichment (slots 5 and 10 in fig. 5). We have noted, however, a specific removal of some components with either the detergent (e.g. the major band 9; slot 5 of fig. 5) or the high salt buffer (e.g. bands no. 3-5; slot 10 in fig. 5) alone. The components of the two major polypeptide bands (bands 2 and 8) constitute a high proportion of the total protein of the residual pore complex-enriched fraction (varying in different experiments from about $40 \%$ to $75 \%$ of the total ratioactivity recovered in gels; fig. 7). In comparative extraction experiments we have observed that simultaneous extraction with a solution containing both Triton X100 and high salt concentration (1 M or 1.5 $\mathrm{M} \mathrm{KCl}$, or $0.5 \mathrm{M} \mathrm{MgCl}_{2}$; $\operatorname{cf}$ [13]) is equally efficient (fig. 5, slots 7 and 8; fig. $7 b$ ). The structures containing the two major components band 2 and band 8 are also resistant to moderate concentrations of urea (fig. 5, slot 11) and guanidinium hydrochloride.

When the polypeptide pattern of the residual pore complex-enriched subfraction

\footnotetext{
$\mathrm{KCl}$ ) and $1 \%$ Triton (slot 3 ). The doubleheaded arrows at the bottom of the gel denote the position of dansylated chymotrypsinogen.
} 


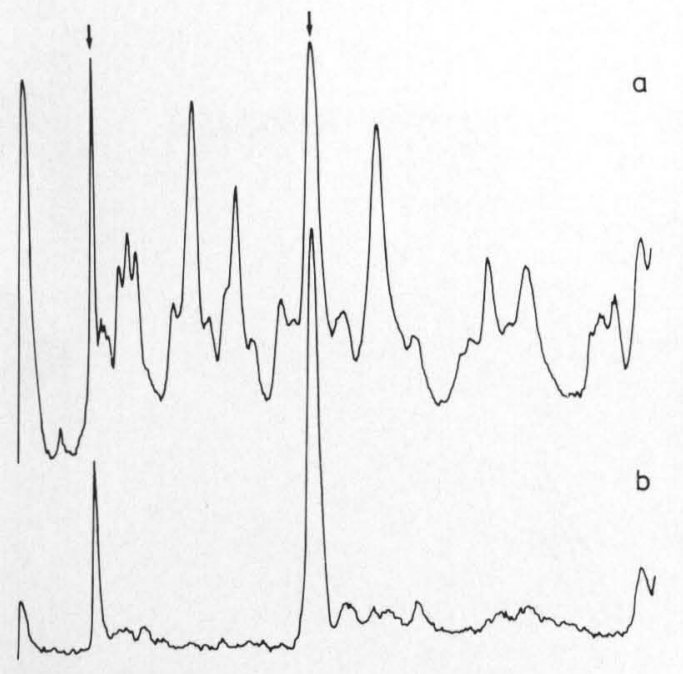

Fig. 7. Specific enrichment of two polypeptides in pore complex-enriched nuclear envelope residues from $X$. laevis oocytes. Representative densitograms of gels shown by fluorography of $\left[{ }^{3} \mathrm{H}\right]$ dansylated polypeptides of nuclear envelopes that are untreated $(a)$ or extracted with high salt buffer and Triton X-100 $(b$; simultaneous extraction) show the enrichment of the two polypeptides with apparent mol. wt of 150000 and 73000 (denoted by arrows) in the pore complexenriched fraction.

of isolated nuclear envelopes is compared with that of the fibrillar residues obtained from total nuclear contents, i.e. after removal of the envelope, in a parallel extraction with high salt concentrations and detergent, a clear difference is noted (fig. 6, slots 1 and 2). Some prominent nucleoplasmic polypeptides, in particular the component comigrating with actin, resist such treatment and are prominent in the nuclear residue (fig. 6, slot 1 ). The obvious absence of significant amounts of the prominent polypeptide components of the extracted pore complex-enriched fraction (fig. 6, slot 2 ) in such residual intranuclear material indicates that they do not merely represent constituents of a fibrillar nuclear matrix framework that are connected to, or might have been dragged along, with the envelope during the preparation.
In the discussion of the enrichment of the two of the major polypeptides of the isolated nuclear envelope the problem of a possible proteolytic cleavage is of importance. The inclusion of a variety of inhibitors of proteolytic enzymes such as phenylmethanesulfonyl fluoride in the isolation media has not resulted in significant changes of the pattern obtained. Moreover, prolonged incubation of the isolated envelopes in the isolation media (varying, for example, from $10 \mathrm{~min}$ to $3 \mathrm{~h}$ in cold " $5: 1$ medium" containing $10 \mathrm{mM} \mathrm{MgCl}_{2}$ ) has likewise not resulted in the breakdown of the major polypeptide components of the pore complex-enriched fraction. In addition, higher concentration polyacrylamide gels of both the non-extracted and the high salt- and detergent-extracted nuclear envelope preparations do not reveal the presence of any considerable amounts of low molecular weight polypeptides or fragments (fig. 6, slot 4). This, taken together with our observation that exogenously added proteases gradually digest the nuclear pore complex structures (data not shown here; cf [43]), concomitant with a dramatic decrease of band 2 and band 8 components suggests that these polypeptides are not exposed to endogenous nuclear envelopebound proteases during the preparation.

When the proteins of isolated nuclear envelopes and the pore complex-enriched residual fractions from maturing oocytes of various urodelan species (see above) are examined by gel electrophoresis, the predominance of two components with mobilities essentially identical with those of bands 2 and 8 of $X$. laevis is generally observed (e.g. fig. 6, slot 3). This similarity in major nuclear envelope components is in contrast, however, to the interspecies differences that are observed in the relative amounts of the major polypeptide compo- 


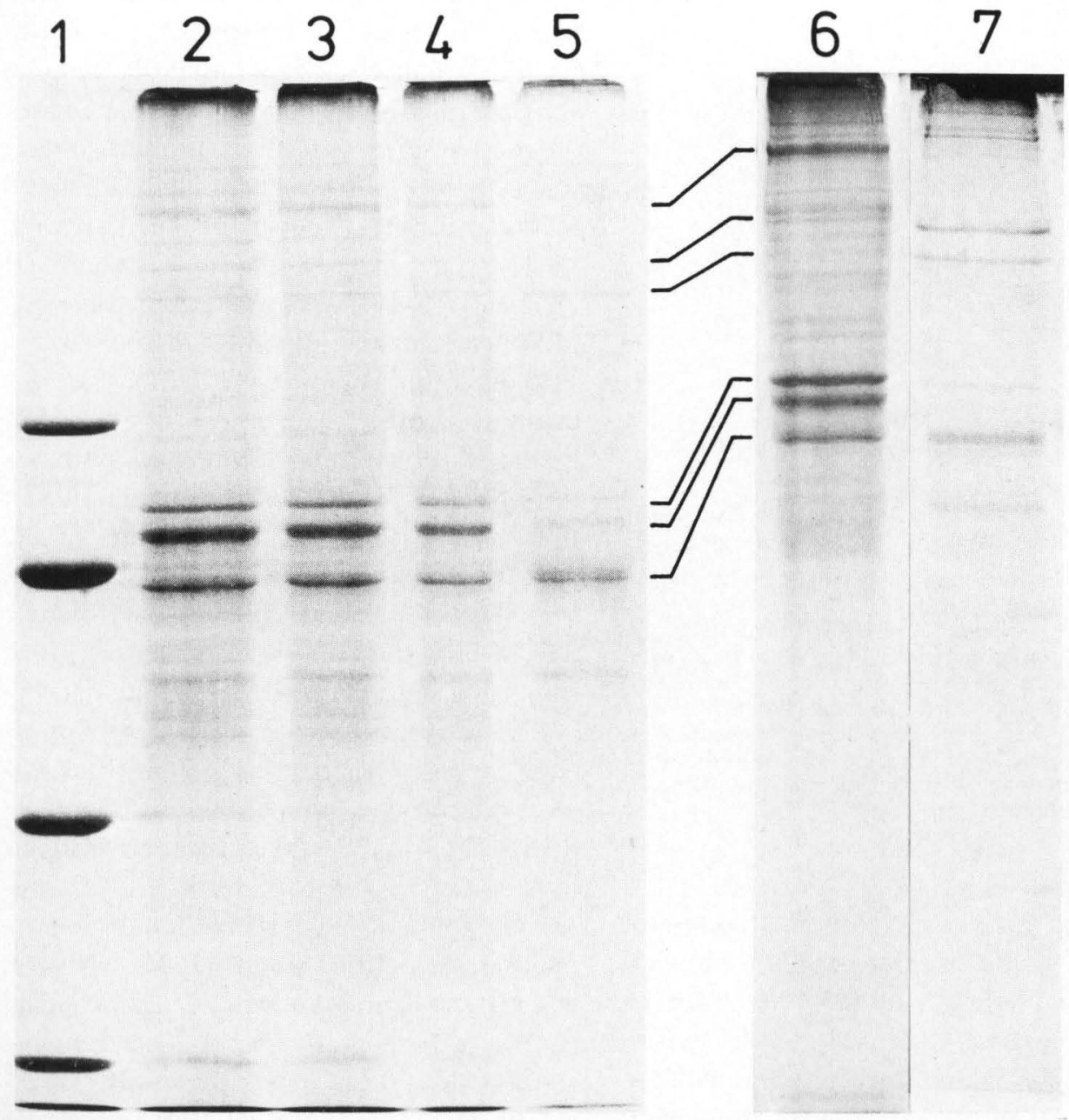

Fig. 8. Polypeptides of pore complex-enriched fractions obtained after extraction of isolated nuclear membranes from rat liver with buffers containing high salt concentrations and Triton X-100, in comparison with non-membranous subfractions derived from the isolated nuclear membranes. Gel electrophoretic separations in SDS-containing polyacrylamide gels $(10 \%$, slots $1-5 ; 12 \%$, slots 6 and 7$)$ of high salt- and Triton X-100 extracted "nuclear membranes purified once" (slots 2 and 3; for designations see Methods) and "nuclear membranes purified twice" (slots 4 and 6) are compared with the pelleted fibrillar subfraction designated "nuclear membrane-derived heavy material" which appears to be enriched in nuclear matrix components (slots 5,7 ). The nuclear pore complexenriched residual fraction from rat liver membranes shows an enrichment of polypeptide bands of apparent mol. wt of 150000 and 73000 (these are the bands denoted by the marks 1 and 5 , from top, between slots 5 and 6 ) which are accompanied by same additional polypeptides, including two prominent ones with apparent mol. wt of about 78000 (mark no. 4) and 66000 (mark no. 6). The latter polypeptide band $(66000 \mathrm{~mol}$. $w t)$, however, is clearly enriched in pellets of material detached from the nuclear membranes during an additional homogenization (slots 5 and 7), together with two minor polypeptide bands of lower electrophoretic mobility (marks 2 and 3 ) thus indicating that it is less tightly associated with the membrane and is probably a nuclear matrix constituent. Slot 1 includes reference proteins (phosphorylase- $a$, bovine serum albumin, actin, chymotrypsinogen). 
nents present in nuclear contents (for example, between $X$. laevis and $T$. alpestris; unpublished data).

\section{Polypeptides of the pore complex- enriched nuclear membrane fractions} from rat liver

The purity and the structural preservation of our nuclei and nuclear membrane fractions isolated from murine liver, including fractions treated with high salt concentrations, has been sufficiently demonstrated in previous publications [for refs, see 5, 6, 8, 28]. When nuclear membrane fractions from rat liver are extracted with both high salt concentrations and detergents, residual structures are obtained which primarily consist of pore complex material, interporous connecting structures ("lamina" sensu ref. [10]; "connecting fibrils" sensu ref. [12]; cf [17]) and probably also adhering fibrous residues from the intranuclear framework. On gel electrophoresis of the polypeptides of such pore complex-enriched residual fractions only few distinct bands are observed, and various authors have emphasized the enrichment of a conspicuous triplet of bands with mobilities corresponding to apparent molecular weights in the range from 62000 to 78000 $([8,10,11,17]$; for bovine liver see ref. [14]). Fig. 8 shows the major polypeptides observed in our pore complex-enriched subfractions from rat liver nuclear membranes ("nuclear membranes purified once") obtained after extraction with high salt buffers and Triton X-100 (for the morphology of these residual fractions cf $[8$, 12]). Five major bands are recognized. The strongest component (varying between 20 and $30 \%$ of the stained bands as estimated from densitograms of a total of 16 analyses) has a relative mobility corresponding to an apparent molecular weight of 73000 . This band is accompanied by two other major components of apparent molecular weights of 78000 and 66000 , and these three together resemble the triplet described by Blobel and co-workers [10, 11]. In addition, we have regularly found a major polypeptide band with an apparent molecular weight of about 150000 (slots 2-6 in fig. 8). A component of similar low electrophoretic mobility has also been noted in such residual fractions by Aaronson \& Blobel [10]. A smaller component (ca 55000 molecular weight; fig. 8, slots 2-4) has been recognized in many, though not in all of our preparations. Low molecular weight components, including histones, are virtually absent (fig. 8; cf [29]).

This polypeptide pattern is also observed when 2-mercaptoethanol or dithiothreitol has been included in all media used during the isolation. It is also essentially unchanged in preparations in which $1 \mathrm{M} \mathrm{KCl}$, $1 \mathrm{M} \mathrm{NaCl}$, or $0.5 \mathrm{M} \mathrm{MgCl}$ has been used, instead of $1.5 \mathrm{M} \mathrm{KCl}$, in the high salt extraction step. Inclusion of various inhibitors of proteolysis likewise has not resulted in considerable alteration of the pattern. Most of the material is solubilized in high concentrations of urea $(6-8 \mathrm{M})$ or guanidinium hydrochloride (4 M) and can be reprecipitated from the solubilized state by dialysis against Tris-buffer ( $10 \mathrm{mM}, \mathrm{pH} 7.2$ ), even in the presence of thiol agents such as $20 \mathrm{mM}$ 2-mercaptoethanol.

The nuclear membrane fraction has further been subfractionated by re-homogenization and centrifugation in a flotation gradient into membrane-enriched material ("nuclear membranes purified twice") and a pellet which contains aggregated fibrils similar to the nuclear matrix preparations (for refs see p. 85). While the detergentextracted nuclear membranes purified twice show the normal pattern (fig. 8, slot 4), 
the pelleted fibrous material separated from the membrane material as well as the fraction designated "first pellet" are characterized by an enrichment of the 66000 molecular weight component and of two minor components of mobilities corresponding to apparent molecular weights of about 115000 and 125000 , concomitant with a reduced proportion of the components of 150000 and 73000 apparent molecular weight (slots 5 and 7 in fig. 8).

\section{DISCUSSION}

The results presented here confirm and extend previous reports that most of the nuclear pore complex material is resistant to treatment with moderately active detergents $[7,10,11,12]$. Resistance of nonmembranous pore complex components to treatments with high salt concentrations and various nucleases, including ribonucleases, as well as a sensitivity to proteases has been repeatedly described [e.g. 6, $28,43,44]$. Such cytochemical observations and our finding that the dry mass of the pore complex does not considerably change during such extractions, together with the results of determinations of the mean contents of pore-associated RNA (41 attograms in maturing $X$. laevis oocytes; [24]), strongly indicate that the bulk of the pore complex material is proteinaceous [ $\mathrm{cf} \mathrm{43,45]} \mathrm{and} \mathrm{is}$ bound by non-ionic forces. This resistant pore complex protein primarily consists of two major polypeptides (apparent molecular weights 150000 and 73000 ), at least in the case of the amphibian oocyte nucleus. The only possible alternative conclusion, namely that these two major polypeptides are not contained in the quantitatively predominant pore complex material but in the pore connecting fibrillar structures, seems very unlikely. Assuming a molar ratio of the two major polypeptide components of $1: 2$ in the native pore complex (see data presented in Results, especially the gel stainings), one can estimate that the average pore complex contains more than thousand of these molecules (about 400 of band 2 component and 800 of band 8 component).

Our results with high salt buffer- and detergent-extracted nuclear membrane preparations from rat liver are compatible with the view that similarly sized polypeptides of about 150000 and 73000 molecular weight are also skeletal components of the pore complex of the hepatocyte. An additional component of about 78000 molecular weight, however, seems to be specific for the preparations made from rat liver. At the moment we cannot decide whether it is located in the pore complex or in the residual interporous structures, or whether it merely reflects the tight association with some residual intranuclear matrix material. As to the component of about 66000 molecular weight observed in the preparations from rat liver, we suggest that most of this band represents an associated intranuclear component, probably of nuclear matrix origin. This tentative conclusion is based on the observed enrichment of this component in non-membranous subfractions separated from the nuclear membrane as well as on the predominance of bands of estimated molecular weights in the 60000-69000 range in total non-histone proteins and in nuclear matrix preparations from liver tissue $[8,14,17]$ as well as from other cells [16, 18-23].

Prominent polypeptide bands with apparent molecular weights in the 65000 80000 range have also been recognized in isolated nuclear membrane fractions from cells with moderate (such as liver) or low (avian erythrocytes) pore frequencies $[10$, 
$18,20,28,46-48]$. It has also been noted that some prominent polypeptides of this size class are present in liver nuclear membrane fractions but not in liver microsomes $[46,47]$. The similarity of the polypeptide patterns of nuclear membrane fractions from avian erythrocytes and from liver cells has led Jackson [18] to conclude "that none of the major polypeptides common to both erythrocyte and liver nuclear membrane are elements of the pore complex". However, the pore frequencies in all the somatic nuclear membranes so far isolated and examined are rather low, relative to that present in oocyte nuclei.

The described resistance properties to treatments with low and high salt concentrations as well as to various detergents adds the nuclear pore complex to the list of membrane-attached structures that are characterized by a specific and localized association with resistant non-membranous elements. Other examples include the plaques of fuzzy coats associated with synaptic function complexes $([49,50]$; see there for further refs), the inner coat of the milk fat globule membranes (for ref. see [51]) and the polygonal coats of coated vesicles (same refs). Thus the major constituents of the pore complex represent a highly symmetrically arranged form of membraneassociated skeletal elements. The observation of at least two major polypeptide bands common to nuclear pore complexenriched fractions from such diverse cells as amphibian oocytes and murine hepatocytes might serve as a basis for the hypothesis that, in all eukaryotic cells, the known universality of nuclear pore complex architecture reflects the involvement of universal polypeptides.

We are indebted to Dr G. F. Bahr and Mr G. F. Engler (Department of Cellular Pathology, Armed Forces Institute of Pathology, Washington, DC, USA) for their help and guidance in the dry weight determinations by quantitative electron microscopy. We thank Miss Stefanie Winter for excellent technical assistance. The work has been partly supported by a grant from the Deutsche Forschungsgemeinschaft.

\section{REFERENCES}

1. Callan, H G \& Tomlin, S G, Proc roy soc, London, ser. B 137 (1950) 367.

2. Franke, W W, Z Zellforsch 105 (1970) 405.

3. Franke, W W \& Scheer, U, J ultrastruct res 30 (1970) 288

4. - Ibid 30 (1970) 317.

5. Franke, W W, Int rev cytol, suppl. 4 (1974) 71.

6. Franke, W W \& Scheer, U, The cell nucleus (ed H Busch) vol. 1, p. 219. Academic Press, New York (1970).

7. - Symp soc exp biol 28 (1974) 249.

8. Franke, W W, Biochem soc symp 42 (1977) 125.

9. Harris, H R, Methodological surveys in biochemistry (ed E Reid) p. 245. Horwood, Chichester (1977).

10. Aaronson, R P \& Blobel, G, Proc natl acad sci US 72 (1975) 1007.

11. Dwyer, N \& Blobel, G, J cell biol 70 (1976) 581 .

12. Scheer, U, Kartenbeck, J, Trendelenburg, M F, Stadler, J \& Franke, W W, J cell biol 69 (1976) 1.

13. Shelton, K R, Biochim biophys acta 455 (1976) 973.

14. Berezney, R \& Coffey, D S, J cell biol 73 (1977) 616.

15. Faiferman, I \& Pogo, O, Biochemistry 14 (1975) 3808 .

16. Riley, E, Keller, J M \& Byers, B, Biochemistry 14 (1975) 3005.

17. Comings, D E \& Okada, A T, Exp cell res 103 (1976) 341.

18. Jackson, R C, Biochemistry 15 (1976) 5641.

19. - Ibid 15 (1976) 5652.

20. Shelton, K R, Cobbs, C S, Povlishock, J T \& Burkat, R K, Arch biochem biophys 174 (1976) 177.

21. Zieve, G \& Penman, S, Cell 8 (1976) 19

22. Hodge, L D, Mancini, P, Davis, F M \& Heywood, P, J cell biol 72 (1977) 194.

23. Wunderlich, F \& Herlan, G, J cell biol 73 (1977) 271.

24. Scheer, U, Z Zellforsch 127 (1972) 127.

25. - Dev biol 30 (1973) 13.

26. Stevens, B J \& André, J, Handbook of molecular cytology (ed A Lima-de-Faria) p. 837. North-Holland, Amsterdam (1969).

27. Colman, A, J embryol exp morphol 32 (1974) 515.

28. Franke, W W, Deumling, B, Ermen, B, Jarasch, E D \& Kleinig, H, J cell biol 46 (1970) 379.

29. Franke, W W, Keenan, T W, Stadler, J, Genz, R, Jarasch, E D \& Kartenbeck, J, Cytobiologie 13 (1976) 28 .

30. Schultz, R M \& Wassarman, P M, Anal biochem 77 (1977) 25.

31. Laemmli, U K, Nature 227 (1970) 680.

32. Thomas, J O \& Kornberg, R D, Proc natl acad sci US 72 (1975) 2626. 
33. Weber, K \& Osborn, M, J biol chem 244 (1969) 4406.

34. Fairbanks, G, Steck, T L \& Wallach, D F H, Biochemistry 10 (1971) 2606.

35. Bonner, M \& Laskey, R A, Eur j biochem 46 (1974) 83.

36. Miller, O L \& Bakken, A H, Acta endocrinol, suppl. 168 (1972) 155.

37. Bahr, G F, Engler, W F \& Mazzone, H M, Quart rev biophys 9 (1976) 459 .

38. Bahr, G F, Micromethods in molecular biology (ed V Neuhoff) p. 257. Springer-Verlag, Heidelberg (1973).

39. Dumont, J N, J morphol 136 (1972) 153.

40. DuPraw, E H, DNA and chromosomes. Holt, Rinehart \& Winston, New York (1970).

41. Merriam, R W \& Hill, R J, J cell biol 69 (1976) 659.

42. De Robertis, E M, Partington, G A, Longthorne, R \& Gurdon, J B, J embryol exp morphol 40 (1977) 199.

43. Koshiba, K, Smetana, K \& Busch, H, Exp cell res 60 (1970) 199.
44. Mentré, P, J microsc 8 (1969) 51.

45. Franke, W W \& Falk, H, Histochemie 24 (1970) 266.

46. Bornens, M \& Kasper, C B, J biol chem 248 (1973) 571 .

47. Kasper, C B, The cell nucleus (ed H Busch) vol. 1, p. 349. Academic Press, New York (1974).

48. Cochran, D L \& Shelton, K R, FEBS lett 71 (1976) 245.

49. Therien, H M \& Mushynski, W E, J cell biol 71 (1976) 807

50. Cohen, R S, Blomberg, F, Berzins, K \& Siekevitz, P, J cell biol 74 (1977) 181 .

51. Franke, W W, Lüder, M R, Kartenbeck, J, Zerban, H \& Keenan, T W, J cell biol 69 (1976) 173.

Received December 30, 1977

Revised version received April 5, 1978

Accepted April 7, 1978 